\title{
On the ellipsoidal statistical model for polyatomic gases
}

\author{
Stéphane Brull*, Jacques Schneider**
}

Pacs numbers: $05.20 . \mathrm{Dd}, 02.60 . \mathrm{Cb}, 02.30 . \mathrm{Xx}, 02.60 . \mathrm{Nm}$

\begin{abstract}
The aim of this article is to construct a $B G K$-type model for polyatomic gases which gives in the hydrodynamic limit the proper transport coefficient. Its construction relies upon a systematic procedure: minimizing Boltzmann entropy under suitable moments constraints $([20,9])$. The obtained model corresponds to the ellipsoidal statistical model introduced in [2]. We also study the return to equilibrium of its solutions in the homogeneous case.
\end{abstract}

*Institut des mathématiques de Toulouse, UMR 5219. Université Paul Sabatier, 118 route de Narbonne, 31062 Toulouse cedex 9, France.

**ANLA, University of Toulon, avenue de l'université, 83957 La Garde, France.

\section{Introduction.}

Classically the monatomic Boltzmann equation is used for describing rarefied gases. But in realistic cases polyatomic gases must be considered. In this case the density function depends on the velocity of particles but also on their internal energy (rotational, vibrational ...) and the density function writes $f=f(t, x, v, I)$ with $(t, x, v, I) \in \mathbb{R}_{+} \times \mathbb{R}^{3} \times \mathbb{R}^{3} \times \mathbb{R}_{+}$. The Boltzmann equation for this density is still

$$
\left(\frac{\partial}{\partial t}+v \cdot \nabla_{x}\right) f=Q(f, f) .
$$

where $Q(f, f)$ is some quadratic collision term which can take different forms $([18,29,8,15])$. But those models are very demanding in computer resources 
(see e.g [4]). Therefore it is of interest to look for simplified models that preserve important properties of the former. The simplest one is the socalled BGK model introduced by Bhatnagar, Gross and Krook ([5]). It writes

$$
R_{B G K}(f)=\rho(\mathcal{M}-f),
$$

where $\mathcal{M}=\mathcal{M}(t, x, v, I)$ is the Maxwellian distribution defined by

$$
\mathcal{M}(v, I)=\frac{\rho \Lambda_{\delta}}{\left(2 \pi R T_{e q}\right)^{3 / 2}\left(R T_{e q}\right)^{\frac{\delta}{2}}} \exp \left(-\frac{|v-u|^{2}}{2 R T_{e q}}-\frac{I^{\frac{2}{\delta}}}{R T_{e q}}\right)
$$

with

$$
\begin{array}{r}
\rho(t, x)=\int_{\mathbb{R}^{3} \times \mathbb{R}_{+}} f d v d I, u(t, x)=\frac{1}{\rho} \int_{\mathbb{R}^{3} \times \mathbb{R}_{+}} v f d v d I, \\
T_{e q}=\frac{2}{(3+\delta) \rho R} \int_{\mathbb{R}^{3} \times \mathbb{R}_{+}}\left(\frac{1}{2}|v-u|^{2}+I^{\frac{2}{\delta}}\right) f d v d I .
\end{array}
$$

$\delta$ is the total number of molecular internal degrees of freedom of the gas and $R=\frac{k}{m}, k$ being the Boltzmann constant and $m$ the molecular mass. Finally

$$
\Lambda_{\delta}^{-1}=\int_{\mathbb{R}_{+}} e^{-I^{\frac{2}{\delta}}} d I
$$

Other important definitions are the total energy $E$

$$
E(t, x)=\int_{\mathbb{R}^{3} \times \mathbb{R}_{+}}\left(\frac{1}{2}|v|^{2}+I^{\frac{2}{\delta}}\right) f d v d I,
$$

and the entropy functional $\mathcal{H}$

$$
\mathcal{H}(f)=\int_{\mathbb{R}^{3} \times \mathbb{R}_{+}} f \ln f d v d I
$$

This model enjoys many important properties of the Boltzmann equation: mass, momentum and energy $E$ are conserved and it is entropy dissipative. But it suffers a deficiency in the hydrodynamical limit at the Navier-Stokes level. Its Prandtl number $\mathrm{Pr}$ is incorrect. Let us recall that $\mathrm{Pr}$ is defined by

$$
\operatorname{Pr}=\frac{\gamma}{\gamma-1} \frac{R \mu}{\kappa}
$$


where $\gamma$ is the ratio of specific heats $\left(\gamma=\frac{C_{p}}{C_{v}}\right)$ which is connected to $\delta$ by the relation

$$
\gamma=\frac{\delta+5}{\delta+3}
$$

$\mu$ and $\kappa$ are respectively the first viscosity and the thermal conductivity. For most gases we have $\operatorname{Pr}<1$ while $\operatorname{Pr}=1$ for the BGK model. It is therefore of interest to look for relaxation models that do not have this drawback. Before proceeding further on let us recall an important fact proved by Toscani ([33]) and Collet ([14]). The definition of $\mathcal{H}$ is not merely the extension to polyatomic gases of the entropy functional for monatomic Boltzmann equation (see [11]) but it is the unique Lyapunov functional for the kinetic equation

$$
\left(\frac{\partial}{\partial t}+v \cdot \nabla_{x}\right) f=\rho(\mathcal{M}-f) .
$$

This is a consequence of a variational principle. Let $C_{f}$ be defined by

$$
C_{f}=\left\{g \geq 0 / \int_{\mathbb{R}^{3} \times \mathbb{R}_{+}}\left(1, v, \frac{1}{2}|v|^{2}+I^{\frac{2}{\delta}}\right) g d v d I=(\rho, \rho u, E)\right\} .
$$

Then there exists a unique (strictly) convex functional -namely $\mathcal{H}$ - such that $\mathcal{M}$ is the (unique) extremal point in $C_{f}$ (remark that conservation laws for the BGK model are equivalent to $\mathcal{M} \in C_{f}$ ). This will explain the choice of entropy $\mathcal{H}$ below. Now the idea is to look for a "new" relaxation term of the form

$$
R(f)=\lambda(G-f),
$$

where $\lambda$ is a parameter and $G$ is a function which are seek so as to obtain in the hydrodynamic limit the correct transport coefficients -the two viscosities $\mu$ and $\alpha \mu$ and thermal conductivity $\kappa$ - and thus the right Prandtl number. $\lambda$ and $G$ depend on macroscopic values of $f$. Moreover $G$ is the extremal point in a set $K_{f}$ of the entropy functional. $K_{f}$ is included in $C_{f}$ to ensure conservation laws and it also contains constraints on other moments $\left(\int m_{i}(v, I) g d v d I\right)_{i}$ of $g$. More precisely

$$
\int m_{i}(v, I) R(f) d v d I=-\lambda_{i} \int m_{i}(v, I) f d v d I, \quad \forall i
$$

where $\left(\lambda_{i}\right)_{i}$ are strictly positive rates. Those constraints are chosen basing on physical considerations: in the hydrodynamic limit each moment 
$\int m_{i}(v, I) f d v d I$ becomes very small and measures in some sense the departure from local equilibrium. Thus $\left(\lambda_{i}\right)_{i}$ are connected to transport coefficients. So the whole procedure consists in

1. choosing the set of moments $\left(m_{i}(v, I)\right)_{i}$ and thus defining $K_{f}$,

2. finding the extremal point $G$ of $K_{f}$ with respect to the entropy,

3. defining all parameters $\lambda$ and $\left(\lambda_{i}\right)_{i}$ by computation of the hydrodynamic limit of (1.7) (with $R(f)$ instead of $R_{B G K}(f)$ ) and comparison with the true Navier-Stokes equation so as to recover the correct transport coefficients.

Entropy minimization principles as well as the idea of using different BGKtype relaxation terms were first considered by Levermore ([20]) in the context of monatomic gases. But in his work the different models could only reach Prandtl numbers larger than 1 . Then one of the author ([30]) has proposed to introduce a single relaxation term $R(f)$ that fits with the collision operator $Q_{\text {Max }}(f, f)$ for monatomic Maxwellian molecules in a weak sense: one adds in $C_{f}$ the constraints that moments of $R(f)$ coincide with those of $Q_{\text {Max }}(f, f)$ up to any chosen order (in this case the fluid limit is "correct"). Finally the approach we present here was proposed by the authors in the case of monatomic gases ([9]) who recovered the classical ellipsoidal statistical model by Holway ([17]).

The paper is divided as follows. In section 2 we recall some physical backgrounds about polyatomic gases. Section 3 is devoted to finding additional moments constraints so as to define $K_{f}$. In section 4 we address the problem of finding the extremal point $G$ in $K_{f}$ with respect to the entropy functional $\mathcal{H}$. Since this problem is subjected to the so-called realizability conditions (see Junk $([25,26])$ and one of the author $([30]))$ we prove that it has a unique solution under necessary and sufficient conditions on the relaxation rates (Theorem 4.1). Then we compute the hydrodynamical limit of the model and set the values of $\left.\lambda,\left(\lambda_{i}\right)_{i}\right)$ by comparison with the true NavierStokes system of equations. This allows to recover the proper transport coefficients and defines entirely (1.9). Moreover (1.9) turns out to be the ES-BGK relaxation model for polyatomic gases introduced in Andries\&al ([2]). In the next section we study the return to equilibrium for the ESBGK model (Theorem 5.1) in the homogeneous case. It is based on a sharp 
estimate of the relative entropy

$$
H(f \mid \mathcal{M})=\int f \ln \left(\frac{f}{\mathcal{M}}\right) d v d I .
$$

Finally this result is illustrated numerically in the case of monatomic gases (section 6). The reason of this simplification is that we want to compare the $L^{1}$-return to equilibrium (and also the relative entropy) between the ESBGK model and the true Boltzmann equation. Those comparisons are not possible in the case of polyatomic gases since there exist only Monte-Carlo methods to solve such problem (except the method by Bahi [4]). We also introduce in this section a new BGK model that allows to recover the proper viscosity for any kind of molecule interaction.

\section{Physical backgrounds.}

We have already defined the macroscopic moments $\rho, u, E$ and $T_{e q}$ in $(1.3,1.5)$. Recall firstly the conservation laws for the BGK model

$$
\int_{\mathbb{R}^{3} \times \mathbb{R}_{+}}\left(1, v, \frac{1}{2}|v|^{2}+I^{\frac{2}{\delta}}\right) \mathcal{M} d v d I=\int_{\mathbb{R}^{3} \times \mathbb{R}_{+}}\left(1, v, \frac{1}{2}|v|^{2}+I^{\frac{2}{\delta}}\right) f d v d I .
$$

Remark that due to mass and momentum conservation the conservation of total energy is equivalent to that of the internal energy

$$
\int_{\mathbb{R}^{3} \times \mathbb{R}_{+}}\left(\frac{1}{2}|c|^{2}+I^{\frac{2}{\delta}}\right) \mathcal{M} d v d I=\int_{\mathbb{R}^{3} \times \mathbb{R}_{+}}\left(\frac{1}{2}|c|^{2}+I^{\frac{2}{\delta}}\right) f d v d I
$$

where $c=v-u$. This definition is equivalent to that of the specific internal energy

$$
e=\frac{1}{\rho} E-\frac{1}{2}|u|^{2}=\frac{3+\delta}{2} R T_{e q} .
$$

$e$ is naturally splitted into two parts $e=e_{t r}+e_{i n t}$ with

$$
e_{t r}=\frac{1}{2 \rho} \int_{\mathbb{R}^{3} \times \mathbb{R}_{+}}|c|^{2} f d v d I, \quad e_{i n t}=\frac{1}{\rho} \int_{\mathbb{R}^{3} \times \mathbb{R}_{+}} I^{\frac{2}{\delta}} f d v d I .
$$

Those energies correspond to the translational and internal energies of the particles. Temperatures are associated to them

$$
T_{t r}=\frac{2}{3 R} e_{t r}, \quad T_{i n t}=\frac{2}{\delta R} e_{i n t} .
$$

Finally the opposite of the stress tensor $\Theta$ is defined by

$$
\Theta=\int_{\mathbb{R}^{3} \times \mathbb{R}_{+}} c \otimes c f d v d I
$$




\section{Setting of the problem.}

The purpose of this paper is to construct a relaxation type term of the form

$$
R(f)=\lambda(G-f),
$$

where parameter $\lambda$ and function $G$ are to be fitted to the proper transport coefficients -the two viscosities $\mu$ and $\mu \alpha$ and thermal conductivity $\kappa$. This requires a particular definition of set of constraints $K_{f}$ which will define $G$ $\left(G \in K_{f}\right)$ through a variational principle. So let us determine $K_{f}$. Remark firstly that macroscopic quantities introduced in the previous section are moments of $f$ related to the polynomial space

$$
\mathcal{P}=\operatorname{span}\left[1, v, v \otimes v, I^{\frac{2}{\delta}}\right] .
$$

We will show in the next section that this polynomial space is well adapted to the variational problem defined below. Secondly conservation laws hold if and only if $G \in K_{f} \subset C_{f}$. That is

$$
\int_{\mathbb{R}^{3} \times \mathbb{R}_{+}}\left(1, v, \frac{1}{2}|v|^{2}+I^{\frac{2}{\delta}}\right) g d v d I=\int_{\mathbb{R}^{3} \times \mathbb{R}_{+}}\left(1, v, \frac{1}{2}|v|^{2}+I^{\frac{2}{\delta}}\right) f d v d I .
$$

Again conservation of total energy can be replaced to that of specific energy using mass and momentum conservation

$$
\int_{\mathbb{R}^{3} \times \mathbb{R}_{+}}\left(\frac{1}{2}|c|^{2}+I^{\frac{2}{\delta}}\right) g d v d I=\int_{\mathbb{R}^{3} \times \mathbb{R}_{+}}\left(\frac{1}{2}|c|^{2}+I^{\frac{2}{\delta}}\right) f d v d I .
$$

Then a mathematical and physical consideration is that $f$ must tend to $\mathcal{M}$ in the homogeneous case. Since $\mathcal{M}$ is isotropic we set an additional constraint

$$
\begin{aligned}
& \int_{\mathbb{R}^{3} \times \mathbb{R}_{+}}\left(c \otimes c-\frac{1}{3}|c|^{2} I d\right) \lambda(g-f) d v d I \\
= & -\lambda_{1} \int_{\mathbb{R}^{3} \times \mathbb{R}_{+}}\left(c \otimes c-\frac{1}{3}|c|^{2} I d\right) f d v d I,
\end{aligned}
$$

where $\lambda_{1}$ is a positive relaxation coefficient. Here $I d$ is the identity matrix in $\mathbb{R}^{3}$. Finally following Borgnakke and Larsen $([6])$ we take into account physical requirement that translational and internal energies tend to the 
specific internal energy $e$

$$
\begin{array}{r}
\int_{\mathbb{R}^{3} \times \mathbb{R}_{+}}\left(\frac{1}{3}|c|^{2}-\frac{2}{3+\delta}\left(\frac{|c|^{2}}{2}+I^{\frac{2}{\delta}}\right)\right) \lambda(g-f) d v d I \\
=-\lambda_{2} \int_{\mathbb{R}^{3} \times \mathbb{R}_{+}}\left(\frac{1}{3}|c|^{2}-\frac{2}{3+\delta}\left(\frac{|c|^{2}}{2}+I^{\frac{2}{\delta}}\right)\right) f d v d I \\
=-\lambda_{2}\left(\int_{\mathbb{R}^{3} \times \mathbb{R}_{+}} \frac{1}{3}|c|^{2} f d v d I-\rho R T_{e q}\right),
\end{array}
$$

where $\lambda_{2}$ is a positive relaxation coefficient (here the last equation is obtained by using the definition of internal energy (2.3)). Now we are able to set our variational problem

"Find $G$ solution to the minimization problem

$$
G=\min _{g \in K_{f}} \mathcal{H}(g)
$$

where $K_{f}$ is the set of functions $g \geq 0$ such that (3.2),(3.4),(3.5) hold."

Remark that only two unknown parameters $\frac{\lambda_{1}}{\lambda}$ and $\frac{\lambda_{2}}{\lambda}$ appear in (3.4) and (3.5) which means that these two relations let us with an additional degree of freedom. Setting $\theta=\frac{\lambda_{2}}{\lambda}$ and using (3.3) constraint (3.5) can be formulated as follows

$$
\int_{\mathbb{R}^{3} \times \mathbb{R}_{+}} \frac{1}{3}|c|^{2} g d v d I=(1-\theta) \int_{\mathbb{R}^{3} \times \mathbb{R}_{+}} \frac{1}{3}|c|^{2} f d v d I+\theta \rho R T_{e q} .
$$

Equation (3.4) can be transformed into

$$
\begin{aligned}
\int_{\mathbb{R}^{3} \times \mathbb{R}_{+}} c \otimes c g d v d I & =\frac{1}{3} \int_{\mathbb{R}^{3} \times \mathbb{R}_{+}}|c|^{2} g d v d I \\
& +\left(1-\frac{\lambda_{1}}{\lambda}\right) \int_{\mathbb{R}^{3} \times \mathbb{R}_{+}}\left(c \otimes c-\frac{1}{3}|c|^{2} I d\right) f d v d I .
\end{aligned}
$$

In order to compare the present work with other works about the ellipsoidal statistical model for polyatomic gases we set

$$
\frac{\lambda_{1}}{\lambda}=1-\nu(1-\theta)
$$

and obtain

$$
\begin{aligned}
\int_{\mathbb{R}^{3} \times \mathbb{R}_{+}} c \otimes c g d v d I & \left.=(1-\theta)\left((1-\nu) \rho R T_{\text {tr }} I d+\nu \Theta\right)\right)+\theta \rho R T_{e q} I d \\
& =\rho \mathcal{T} .
\end{aligned}
$$

This means that this tensor is defined entirely in terms of macroscopic quantities depending only on $f$. This will make easier the computation of $G$. 


\section{Construction of the relaxation model.}

The aim of this section is to construct the relaxation model. It is performed in three steps.

1. Derive necessary and sufficient conditions on the values of $\theta$ and $\nu$ such that (3.6) possesses a solution. Give the shape of its solution $G$.

2. Define relaxation rates $\lambda_{1}, \lambda_{2}$ and $\lambda$ such as to recover proper transport coefficients in the hydrodynamic limit up to Navier-Stokes level.

3. Comparison with the ellipsoidal statistical model for polyatomic gases ([2]).

\subsection{Solution to the minimization problem (3.6).}

Our first concern is to solve minimization problem (3.6). Let us recall that such a minimization problem is subjected to the so-called realizability condition. For this problem we refer to the papers by Junk ([26]) and Schneider ([30]). A natural condition for realizability is $K_{f} \neq \emptyset$.

Lemma 4.1. Let $\chi$ be a symmetric definite positive tensor of order two and consider the set $K$ of functions $g \geq 0$ s.t.

$$
\begin{aligned}
\int g d v d I=\rho, \quad \frac{1}{\rho} \int v g d v d I=u, & \frac{\delta}{2 R \rho} \int|v-u|^{2} g d v d I=T, \\
\int\left(\frac{|v-u|^{2}}{2}+I^{\frac{2}{\delta}}\right) g d v d I & =E
\end{aligned}
$$

for some $(\rho, u, T, E)$ in $\mathbb{R}_{+}^{*} \times \mathbb{R}^{3} \times \mathbb{R}_{+}^{*} \times \mathbb{R}_{+}^{*}$ with $E>2 R \rho$ and

$$
\frac{1}{\rho} \int_{\mathbb{R}^{3}} c \otimes c g d v d I=\chi
$$

Then $K$ is not empty.

Proof. The proof is given in appendix A.

It is clear that condition " $\chi$ is symmetric definite positive" in the above lemma imposes conditions on $\nu$ and $\theta$ in the minimization problem. As we are going to see those those conditions are also sufficient to ensure that the problem (3.6) possesses a solution for each density function $f$ in the weighted $L^{1}$-space

$$
L_{2}^{1}=\left\{f /\left(1+|v|^{2}+I^{\frac{2}{\delta}}\right) f \in L^{1}\right\} .
$$


Theorem 4.1. Let $f(f \neq 0)$ be a nonnegative function in $L_{2}^{1}, \nu \in\left[-\frac{1}{2}, 1[\right.$ and $\theta \in[0,1]$. Then minimization problem (3.6) admits a unique solution $G$ defined by

$$
G(v, I)=\frac{\rho \Lambda_{\delta}}{\sqrt{\operatorname{det}(2 \pi \mathcal{T})}\left(R T_{e q}\right)^{\frac{\delta}{2}}} \exp \left(-\frac{1}{2}\left\langle c, \mathcal{T}^{-1} c\right\rangle-\frac{I^{\frac{2}{\delta}}}{R T_{r e l}}\right) .
$$

where

$$
T_{r e l}=\theta T_{e q}+(1-\theta) T_{i n t}
$$

Conversely if $K_{f} \neq \emptyset$ for all nonnegative functions $f \in L_{2}^{1}(f \neq 0)$ then $\nu \in$ $\left[-\frac{1}{2}, 1[, \theta \in[0,1]\right.$ and (3.6) admits a unique solution for each nonnegative function in $L_{2}^{1}$.

Proof. (Theorem 4.1.)

Step 1: It is easy to see that

$$
\nu \in\left[-\frac{1}{2}, 1\left[\text { and } \theta \in[0,1] \Rightarrow \mathcal{T} \in S_{3}^{+}(\mathbb{R}),\right.\right.
$$

therefore $K_{f} \neq \emptyset$ according to the above lemma. Let now $\mathbf{m}(v, I)$ be a generating vector basis of $\mathcal{P}$ and consider the set $\Lambda$ defined with

$$
\Lambda=\left\{\eta \in \mathbb{R}^{11} / \exp (\eta \cdot \mathbf{m}(v, I)) \in L^{1}\right\} .
$$

Then $\lambda \neq \emptyset$ and $\lambda \cap \partial \lambda=\emptyset$. This can be readily checked by writing any polynomial $p(v, I) \in \mathcal{P}$ as

$$
p(v, I)=a+b \cdot v+v^{T} \cdot A \cdot v+c I^{\frac{2}{\delta}},
$$

with $(a, b, A, c) \in \mathbb{R} \times \mathbb{R}^{3} \times S_{3}(\mathbb{R}) \times \mathbb{R}$. Then $\exp (p(v, I)) \in L^{1}\left(\mathbb{R}^{3} \times \mathbb{R}\right.$ if and only if $-A \in S_{3}^{+}(\mathbb{R})$ and $c<0$. Then according to [26] there exists a unique solution to (3.6) which is of the form $G(v)=\exp (m(v, I))$ with $m(v, I) \in \mathbf{P}$. In this particular case the solution $G$ can be computed explicitly using (3.2) and (3.8). Remark that this first step can also be obtained in a similar way using Theorem 2 in [30].

Step 2: In the sequel we set $\rho=1$ and $R=1$ for the sake of clarity.

Let $f$ be a nonnegative function $(f \neq 0)$ in $L_{2}^{1}$ and define $T_{t r}$ and $T_{e q}$ by (2.4), (2.3). Then $K_{f} \neq \emptyset$ implies (see (3.7))

$$
0<\int_{\mathbb{R}^{3} \times \mathbb{R}_{+}} \frac{1}{3}|c|^{2} g d v d I=(1-\theta) T_{t r}+\theta T_{e q}
$$


It is always possible to find a sequence of nonnegative functions $f_{n} \in L_{2}^{1}$ such that

$$
T_{t r}^{n}=\frac{1}{3} \int|c|^{2} f^{n} d v d I=\frac{1}{3} f_{c}^{n} \rightarrow 0 \text { as } n \rightarrow \infty
$$

while

$$
T_{e q}^{n}=T_{e q}=\frac{2}{3+\delta} \int\left(\frac{|c|^{2}}{2}+I^{\frac{2}{\delta}}\right) f^{n} d v d I=\frac{2}{3+\delta}\left(\frac{1}{2} f_{c}^{n}+f_{I}^{n}\right)
$$

is fixed. Then (4.3) writes

$$
T_{t r}^{n}+\theta\left(T_{e q}-T_{t r}^{n}\right)=\frac{1}{3} f_{c}^{n}+\theta\left(\frac{2}{3+\delta}\left(\frac{f_{c}^{n}}{2}+f_{I}^{n}\right)-\frac{1}{3} f_{c}^{n}\right)>0 .
$$

Suppose now that $\theta=1+\varepsilon>1$ for small $\varepsilon$ then

$$
2(1+\varepsilon) f_{I}^{n}<\left(1-\frac{\varepsilon \delta}{3}\right) f_{c}^{n}
$$

which is not possible for $n$ big enough. Hence $\theta \leq 1$.

Now let us consider constraint (3.8) and denote with $\left(\Theta_{i}\right)_{i=1 \ldots 3}$ the eigenvalues of $\Theta$ and with $\left(\tau_{i}\right)_{i=1 \ldots 3}$ the eigenvalues of $\mathcal{T}$. On one side one has

$$
\left.\Theta_{i} \in\right] 0,3 T_{t r}[, \quad \forall i=1 \ldots 3
$$

and on the other side $\tau_{i}>0, \forall=1 \ldots 3$. It is clear that $\Theta$ and $\mathcal{T}$ can be diagonalized in the same basis so that (3.8) leads to

$$
(1-\theta)\left((1-\nu) T_{t r}+\nu \Theta_{i}\right)+\theta T_{e q}>0, \quad \forall i=1 \ldots 3 .
$$

It is always possible to find a sequence of functions $\left(f_{n}\right)$ such that $T_{t r}^{n}=T_{t r}>0$ is fixed while for a given index $i$ one has $\Theta_{i}^{n} \rightarrow 0$. Hence by taking $\theta=0$ in the previous equation we get $(1-\nu) T_{t r}>0$ and thus $1<\nu$. In the same way letting $\Theta_{i}^{n}$ tend to $3 T_{t r}$ we get $(1+2 \nu) T_{t r} \geq 0$ and so $\nu \geq-\frac{1}{2}$.

Remark 1. The maximal degree 2 (in $v)$ of $\mathcal{P}$ ensures existence and uniqueness of the solution to minimization problem (3.6). A maximal degree of order 3 would remove this result. But $\mathcal{P}$ seems not well adapted to the requirement of finding right thermal conductivity $\kappa$ since it involves moment of density function of degree 3 . Nevertheless we will show in the next section that constraints related to $\mathcal{P}$ are sufficient to recover at the hydrodynamic level Navier-Stokes equations with proper transport coefficients.

Remark 2. This model is entropic as proved by Andries Eal ([2]). This can also be obtained by generalizing the proof given in [9]. 


\subsection{Definition of $\lambda_{1}, \lambda_{2}$ and $\lambda$.}

In this part we determine the hydrodynamic limit of the equation

$$
\left(\frac{\partial}{\partial t}+v \cdot \nabla_{x}\right) f=\frac{\lambda}{\varepsilon}(G-f)
$$

so as to recover the Navier-Stokes transport with proper transport coefficients -the two viscosities $\alpha$ and $\alpha \mu$ and the thermal conductivity $\kappa$. This will define relaxation coefficients $\lambda_{1}, \lambda_{2}$ and $\lambda$. Here $\varepsilon$ is the Knudsen number defined as the ratio between the mean free path and a typical length scale (see [11] for a discussion about this nondimensional number). Recall that the Navier-Stokes system of equations writes

$$
\partial_{t}\left(\begin{array}{c}
\rho \\
\rho u_{i} \\
E
\end{array}\right)+\partial_{x_{j}} \cdot\left(\begin{array}{c}
\rho u_{j} \\
\rho u_{i} u_{j}+P \delta_{i j} \\
E u_{j}+P u_{j}
\end{array}\right)=\varepsilon \partial_{x_{j}} \cdot\left(\begin{array}{c}
0 \\
\sigma_{i j} \\
u_{i} \sigma_{i j}+\kappa \partial_{x_{j}} T
\end{array}\right)
$$

where the pressure is given by the polytropic law

$$
P=\rho R T=(\gamma-1) \rho e,
$$

total energy by

$$
E=\frac{1}{2} \rho|u|^{2}+\rho e
$$

and viscosity tensor by

$$
\sigma_{i j}=\mu\left(\partial_{x_{j}} u_{i}+\partial_{x_{i}} u_{j}-\alpha \operatorname{div}(u) \delta_{i j}\right) .
$$

The hydrodynamic limit of (4.5) can be obtained using the classical ChapmanEnskog expansion. This consists in letting $\varepsilon \rightarrow 0$ and expanding $f$ with respect to $\varepsilon$

$$
f=f^{(0)}+\varepsilon f^{(1)}+\varepsilon^{2} f^{(2)}+\ldots
$$

Since $G$ depends on $f$ one also has

$$
G=G^{(0)}+\varepsilon G^{(1)}+\varepsilon^{2} G^{(2)}+\ldots,
$$

where $G^{(i)}$ are functions of moments of $\left(f^{(j)}\right)_{j=1 \ldots i} . f^{(0)}=G^{(0)}$ is a Maxwellian distribution as a consequence of $\mathrm{H}$-theorem (remark 1). Assuming that this function has the same first moments as $f(t, x, v, I)$ then $f^{(0)}=G^{(0)}=\mathcal{M}(t, x, v, I)$ and the following orthogonality relations hold

$$
\int\left(1, v, \frac{1}{2}|c|^{2}+I^{\frac{2}{\delta}}\right) f^{(1)} d v d I=\int\left(1, v, \frac{1}{2}|c|^{2}+I^{\frac{2}{\delta}}\right) G^{(1)} d v d I=0 .
$$


Here equations on internal energies are obtained using the same argument as for equation (3.3). Then we are going to determine $\lambda_{1}, \lambda_{2}$ and $\lambda$ by using the set of constraints $(3.2),(3.5),(3.4)$ and equation (4.5) at order 0

$$
\left(\frac{\partial}{\partial t}+v \cdot \nabla_{x}\right) \mathcal{M}=\lambda\left(G^{(1)}-f^{(1)}\right) .
$$

Multiplying this equation with $\left(1, v, \frac{1}{2}|v|^{2}+I^{\frac{2}{\delta}}\right)$ and integrating it over $v$ and $I$ gives the Euler system of equations. This system is used to compute the left-hand side of (4.9) only in terms of space derivatives (see e.g [19])

$$
\begin{aligned}
\left(\frac{\partial}{\partial t}\right. & \left.+v \cdot \nabla_{x}\right) \mathcal{M}=\left(c\left(\frac{c^{2}+2 I^{\frac{2}{\delta}}}{2 R T_{e q}}-\frac{5+\delta}{2}\right) \cdot \nabla\left(\ln T_{e q}\right)\right. \\
& +\frac{1}{R T_{e q}}\left(\left(c \otimes c-\frac{1}{3+\delta}\left(|c|^{2}+2 I^{\frac{2}{\delta}}\right) I d\right): D(u)\right) \mathcal{M}
\end{aligned}
$$

where $D(u)$ is defined with

$$
D(u)=\frac{1}{2}\left(\nabla_{x} u+\nabla_{x} u^{t}\right)-\frac{1}{3} \operatorname{div}(u) I d .
$$

Then

$$
\int v_{i} v_{j}\left(\frac{\partial}{\partial t}+v \cdot \nabla_{x}\right) \mathcal{M} d v d I=P\left(\frac{\partial u_{i}}{\partial x_{j}}+\frac{\partial u_{j}}{\partial x_{i}}-\frac{2}{3+\delta} \operatorname{div}(u) \delta_{i j}\right) .
$$

Here

$$
P=\rho R T_{e q}
$$

since the Euler system of equations yields $T_{e q}=T$. Now expanding relation (3.5) at order 1 (with $G$ instead of $g$ ) and using (4.9) one finds that the trace of $\Theta^{(1)}$ satisfies the relations

$$
\begin{aligned}
\int|c|^{2} f^{(1)} d v d I & =-\frac{1}{\lambda_{2}} \int|c|^{2}\left(\frac{\partial}{\partial t}+v \cdot \nabla_{x}\right) \mathcal{M} d v d I \\
& =-\frac{1}{\lambda_{2}} \int|v|^{2}\left(\frac{\partial}{\partial t}+v \cdot \nabla_{x}\right) \mathcal{M} d v d I
\end{aligned}
$$

Here last equation is obtained by using orthogonality relations (4.8) together with (4.9). Comparing this with (4.11) gives on one hand

$$
\int|c|^{2} f^{(1)} d v d I=-\frac{P}{\lambda_{2}} \frac{2}{3+\delta} \operatorname{div}(u)
$$


But on the other hand the trace of $-\Theta^{(1)}$ must be equal to that of stress tensor $\sigma_{i j}$

$$
\mu(2-3 \alpha) \operatorname{div}(u)
$$

So that

$$
\lambda_{2}=\frac{P}{\mu} \frac{2}{3+\delta} \frac{1}{2-3 \alpha} .
$$

Correspondingly using relation (3.4), the traceless part of $-\Theta^{(1)}$

$$
\begin{aligned}
-\int c_{i} c_{j} f^{(1)} d v d I & =\frac{1}{\lambda_{1}} \int c_{i} c_{j}\left(\frac{\partial}{\partial t}+v \cdot \nabla_{x}\right) \mathcal{M} d v d I \\
& =-\frac{P}{\lambda_{1}}\left(\frac{\partial u_{i}}{\partial x_{j}}+\frac{\partial u_{j}}{\partial x_{i}}\right) i \neq j
\end{aligned}
$$

must equate the traceless part of stress tensor $\sigma_{i j}$ so that

$$
\lambda_{1}=\frac{P}{\mu} .
$$

Finally notice that the first polynomials in the right hand side of equation (4.10) are nothing but the Hermite polynomials $H_{i}^{3}(c, I)$ with respect to $\mathcal{M}$. Hence they are orthogonal to $\mathcal{P}$ for the scalar product $\int \phi \psi \mathcal{M} d v d I$. This makes easier the computation of

$$
\begin{array}{r}
\int H_{i}^{3}(c, I)\left(G^{(1)}-f^{(1)} d v d I=-\frac{1}{\lambda} \int H_{i}^{3}(c, I)\left(\frac{\partial}{\partial t}+v \cdot \nabla_{x}\right) \mathcal{M} d v d I\right. \\
=-\frac{1}{\lambda}\left[P u_{i}\left(\frac{\partial u_{i}}{\partial x_{j}}+\frac{\partial u_{j}}{\partial x_{i}}-\frac{2}{3+\delta} \operatorname{div}(u) \delta_{i j}\right)+\frac{5+\delta}{2 \lambda} P R \partial_{x_{i}} T\right]
\end{array}
$$

where we the last equation is deduced from (4.10). On the other hand comparing this expression with the Navier-Stokes term for thermal conductivity one obtains

$$
\lambda=\frac{5+\delta}{2} \frac{P R}{\kappa} .
$$

This last definition together with (4.13),(4.14) define entirely the relaxation model (1.9). 


\subsection{Comparison with the ellipsoidal statistical model}

Let us recall (see (3.7)) that

$$
\nu=\frac{1}{1-\theta}\left(1-\frac{\lambda_{1}}{\lambda}\right) .
$$

Therefore using (4.14)

$$
\lambda=\frac{\lambda_{1}}{1-\theta \nu+\nu}=\frac{P}{\mu(1-\nu+\theta \nu)}
$$

so that $R(f)$ also writes

$$
R(f)=\frac{P}{\mu(1-\nu+\theta \nu)}(G-f)
$$

This turns out to be the ellipsoidal statistical model found in [2] for polyatomic gases.

Remark 3. The Prandtl number of this model is

$$
\operatorname{Pr}=\frac{5+\delta}{2} \frac{R \mu}{\kappa}=\frac{\lambda}{\lambda_{1}}=\frac{1}{1-\nu+\theta \nu} .
$$

For example, in the situation of diatomic gases we have the experimental values $\operatorname{Pr}=\frac{5}{7}$ and $\theta=\frac{1}{5}$. Hence $\nu=-\frac{1}{2}$.

\section{Return to equilibrium.}

We consider in this part the behavior of the solution to the space homogeneous equation

$$
\frac{\partial}{\partial t} f=\lambda(G-f)
$$

when $t$ tends to infinity. We show that the relaxation rates $\lambda_{1}, \lambda_{2}$ have still an influence on the asymptotic behavior of $f$.

The idea is to derive a rate on the decay of relative entropy $H(f \mid G)(1.10)$ together with a Gronwall lemma. The main result of this section is the following theorem.

Theorem 5.1. For $\lambda_{1}$ and $\lambda_{2}$ defined in (4.14) and (4.13) let us put $\lambda_{3}=\min \left(\lambda_{1}, \lambda_{2}\right)$. Then for all $f$ solution to (5.1), we have

$$
\|f-\mathcal{M}\|_{L^{1} \mathbb{R}^{3} \times \mathbb{R}_{+}}(t)=\mathcal{O}\left(e^{-\lambda_{3} t}\right) .
$$

where $\mathcal{M}$ has the same definition as usual (1.2) dropping out the space dependance. 
Proof. By derivation of the relative entropy (1.10) it holds that

$$
\frac{\partial}{\partial t}\left(f \ln \frac{f}{G}\right)=\left(\frac{\partial}{\partial t} f\right) \ln \frac{f}{G}+\left(\frac{\partial}{\partial t} f\right)-f \frac{\frac{\partial}{\partial t} G}{G} .
$$

We have two cases: $\theta=0$ and $0<\theta \leq 1$. We choose to treat the second case which is more complicated. Let us first show that

$$
\left\|\frac{\partial_{t} G}{G}\right\|_{\infty}=\mathcal{O}\left(e^{-\lambda_{3} t}\right)
$$

Let $\left(\tau_{1}, \tau_{2}, \tau_{3}\right)$ (resp. $\left.\Theta_{1}, \Theta_{2}, \Theta_{3}\right)$ be the eigenvalues of $\mathcal{T}$ (resp. $\Theta$ ). Then $\frac{\frac{\partial}{\partial t} G}{G}$ has the expression

$$
\frac{\frac{\partial}{\partial t} G}{G}=\frac{2}{\delta} \frac{\frac{\partial}{\partial t} T_{r e l}}{T_{r e l}}+\frac{1}{2}\left(\frac{\tau_{1}^{\prime}}{\tau_{1}}+\frac{\tau_{2}^{\prime}}{\tau_{2}}+\frac{\tau_{3}^{\prime}}{\tau_{3}}\right)+\left(\left\langle\frac{1}{2} c, \frac{\partial}{\partial t}\left(\mathcal{T}^{-1}\right) c\right\rangle-I^{\frac{2}{\delta}} \frac{\partial}{\partial t}\left(\frac{1}{T_{r e l}}\right)\right) .
$$

Using (3.5) we obtain after multiplication of (5.1) by $\frac{1}{3}|c|^{2}-\frac{2}{3+\delta}\left(\frac{|c|^{2}}{2}+I^{\frac{2}{\delta}}\right)$ and integration

$$
\left(T_{t r}-T_{e q}\right)^{\prime}=-\lambda_{2}\left(T_{t r}-T_{e q}\right)
$$

So

$$
T_{t r}-T_{e q}=\mathcal{O}\left(e^{-\lambda_{2} t}\right) .
$$

On the other side multiplying the equation (5.1) by $c \otimes c-\frac{1}{3}|c|^{2} I d$ and integrating on $\mathbb{R}^{3} \times \mathbb{R}_{+}$we have according to (3.4)

$$
\left(\Theta-R T_{t r} I d\right)^{\prime}=-\lambda_{1}\left(\Theta-R T_{t r} I d\right) .
$$

Therefore $\Theta(t)$ satisfies

$$
\left(\Theta-R T_{t r} I d\right)=\mathcal{O}\left(e^{-\lambda_{1} t}\right) .
$$

The symmetric tensor $\Theta(0)$ can be diagonalized as $\Theta(0)=P^{t} \Delta(0) P$ where $\Delta(0)$ is the diagonal matrix whose terms are $\left(\theta_{1}(0), \theta_{2}(0), \theta_{3}(0)\right)$.

From to the relations $(3.8,5.7), \mathcal{T}(t)$ can be diagonalized in the same basis as $\Theta(t)$ and $\Theta(0)$. Then $\mathcal{T}(t)=P^{t} D(t) P$, where $D$ is the diagonal matrix whose terms are $\left(\tau_{1}, \tau_{2}, \tau_{3}\right)$. Therefore

$$
\tau_{i}=(1-\theta)\left((1-\nu) R T_{t r}+\nu \Theta_{i}\right)+\theta R T_{e q}
$$


and

$$
\frac{\tau_{i}^{\prime}}{\tau_{i}}=\frac{(1-\theta)\left((1-\nu) \lambda\left(T_{e q}-T_{t r}\right) I d+\lambda_{1} \nu\left(T_{t r}-\Theta_{i}\right)\right)}{(1-\theta)\left((1-\nu) T_{t r}+\nu \Theta_{i}\right)+\theta T_{e q}} .
$$

As $\theta \neq 0$ by putting $\lambda_{3}=\min \left(\lambda_{1}, \lambda_{2}\right)$ and by using $(5.5,5.7)$, it holds that

$$
\frac{\tau_{i}^{\prime}}{\tau_{i}}=\mathcal{O}\left(e^{-\lambda_{3} t}\right)
$$

As

$$
\frac{\partial}{\partial t}\left(\frac{1}{T_{r e l}^{\frac{2}{\delta}}}\right)=\frac{2}{\delta} \frac{T_{r e l}^{\prime}}{T_{r e l}^{\frac{2}{\delta}+1}}
$$

and $T_{r e l}^{\prime}=(1-\theta) T_{i n t}^{\prime}$, it holds that

$$
\frac{\partial}{\partial t}\left(\frac{1}{T_{\text {rel }}^{\frac{2}{\delta}}}\right)=\frac{2}{3}(1-\theta) \lambda_{2} \frac{T_{e q}-T_{t r}}{T_{\text {rel }}^{\frac{2}{\delta}}} .
$$

Hence from (5.5)

$$
\frac{\partial}{\partial t}\left(\frac{1}{T_{r e l}^{\frac{2}{\delta}}}\right)=\mathcal{O}\left(e^{-\lambda_{2} t}\right)
$$

Moreover

$$
\left\langle c, \frac{\partial}{\partial t}\left(\mathcal{T}^{-1}\right) c\right\rangle=\left\langle c, \frac{\partial}{\partial t}\left(D^{-1}\right) c\right\rangle=-\sum_{i=1}^{3} \frac{\tau_{i}^{\prime}}{\tau_{i}^{2}}\left|c_{i}\right|^{2} .
$$

Therefore

$$
\frac{\frac{\partial}{\partial t} G}{G}=\frac{T_{r e l}^{\prime}}{T_{r e l}}\left(-\frac{2}{\delta}+\frac{I^{\frac{2}{\delta}}}{T_{r e l}^{\delta}}\right)-\frac{1}{2} \sum_{i=1}^{3} \frac{\tau_{i}^{\prime}}{\tau_{i}}\left(1+\frac{\left|c_{i}\right|^{2}}{\tau_{i}}\right)
$$

and (5.3) follows.

Now let us finish the proof of Theorem 5.1. By integrating (5.1) and by using mass conservation, it holds that

$$
\frac{\partial}{\partial t}\left(\int_{\mathbb{R}^{3}} f \ln \left(\frac{f}{G}\right) d v\right)=\lambda \int_{\mathbb{R}^{3}}(G-f) \ln \left(\frac{f}{G}\right) d v-\int_{\mathbb{R}^{3}} f \frac{\frac{\partial}{\partial t} G}{G} d v .
$$


As $G \ln \left(\frac{f}{G}\right) \leq 0$, it holds that

$$
\frac{\partial}{\partial t} H(f \mid G) \leq-\lambda H(f \mid G)+h(t)
$$

with

$$
\begin{array}{r}
h(t)=-\int_{\mathbb{R}^{3} \times \mathbb{R}_{+}} f\left(\left(-\frac{2}{\delta}+\frac{I^{\frac{2}{\delta}}}{T_{r e l}^{\delta}}\right) \frac{2}{3}(1-\theta) \lambda_{2} \frac{T_{e q}-T_{t r}}{T_{r e l}}\right) \\
\left.-\frac{1}{2} \sum_{i=1}^{3}\left(1+\frac{\left|c_{i}\right|^{2}}{\tau_{i}} \frac{(1-\theta)\left((1-\nu) \lambda\left(T_{e q}-T_{t r}\right)+\lambda_{1} \nu\left(T_{t r}-\Theta_{i}\right)\right)}{(1-\theta)\left((1-\nu) T_{t r}+\nu \Theta_{i}\right)+\theta T_{e q}}\right)\right) d v d I .
\end{array}
$$

So the relative entropy $H(f \mid G)$ satisfies the inequality

$$
\frac{\partial}{\partial t} H(f \mid G)(t) \leq-\lambda(H(f \mid G)(t)-H(f \mid G)(0))+h(t)-\lambda H(f \mid G)(0) .
$$

According to Gronwall lemma it comes that

$$
\begin{aligned}
H(f \mid G)(t) & \leq h(t)-(1-t \lambda H(f \mid G)(0))+\int_{0}^{t} h(s) d s \\
& -\int_{0}^{t} \int_{0}^{u} h(s) d s h(s) e^{-\lambda s} d u .
\end{aligned}
$$

Hence as $H(f \mid G)(0) \geq 0$, we get for $t$ big enough

$$
H(f \mid G)(t) \leq h(t)+\int_{0}^{t} h(s) d s-\int_{0}^{t} \int_{0}^{u} h(s) d s e^{-\lambda s} d u .
$$

According to Lemma 5.3, $h$ satisfies

$$
\|h\|_{\infty}=\mathcal{O}\left(e^{-\lambda_{3} t}\right) .
$$

Therefore $H(f \mid G)$ satisfies the estimate

$$
H(f \mid G)=\mathcal{O}\left(e^{-\lambda_{3} t}\right) .
$$

Therefore from Czsisar-Kullback ([13]) inequality we get

$$
2 H(f \mid G) \geq \frac{1}{\rho}\|f-G\|_{L^{1}}^{2}(t)
$$

(the factor $\rho$ is added because of the normalization) and the estimate (5.9) gives

$$
\|f-G\|_{L^{1}}^{2}(t)=\mathcal{O}\left(e^{-\lambda_{3} t}\right) .
$$


Moreover in order to estimate $\|G-M\|_{L^{1}}(t)$, we consider the relative entropy

$$
H(G \mid M)=\int_{\mathbb{R}^{3}} G \ln \left(\frac{G}{M}\right) d v d I .
$$

By using the expressions of $G$ and $M, H(G \mid M)$ has the expression

$$
H(G \mid M)=\int G\left(\ln \left(\frac{\left(R T_{e q}\right)^{\frac{3}{2}}}{\sqrt{\operatorname{det}(\tau)}}\right)+\frac{|c|^{2}}{2 R T_{e q}}-\frac{1}{2}\left\langle c, \tau^{-1} c\right\rangle+\frac{I^{\frac{2}{\delta}}}{R}\left(\frac{1}{T_{e q}}-\frac{1}{T_{r e l}}\right)\right) d v .
$$

The second term of (5.12) can be computed as follows

$$
\begin{aligned}
\frac{|c|^{2}}{2 R T_{e q}}-\frac{1}{2}\left\langle c, \tau^{-1} c\right\rangle & =\sum_{i=1}^{3} \frac{c_{i}^{2}}{2 R}\left(\frac{1}{T_{e q}}-\frac{1}{\tau_{i}}\right) \\
& =\sum_{i=1}^{3} \frac{c_{i}^{2}}{2 R T_{e q} \tau_{i}}\left(\tau_{i}-T_{e q}\right)=\mathcal{O}\left(e^{-\lambda_{3} t}\right) .
\end{aligned}
$$

According to the definition of $T_{r e l}, T_{e q}$ and of (5.5)

$$
\frac{1}{T_{e q}}-\frac{1}{T_{r e l}}=\mathcal{O}\left(e^{-\lambda_{3} t}\right) \text {. }
$$

In order to estimate $\ln \left(\frac{\left(R T_{e q}\right)^{\frac{3}{2}}}{\sqrt{\operatorname{det}(\tau)}}\right)$, we use that $\theta_{i}(t)$ has the expression $\theta_{i}(t)=A e^{-\lambda_{1} t}+R T_{t r}$ and $T_{e q}$ has the expression $T_{e q}=T_{t r}+B e^{-\lambda_{2} t}$. Hence we get

$$
\ln \left(\frac{\left(R T_{e q}\right)^{\frac{3}{2}}}{\sqrt{\operatorname{det}(\tau)}}\right)=\mathcal{O}\left(e^{-\lambda_{3} t}\right)
$$

Therefore

$$
H(G \mid M)=\mathcal{O}\left(e^{-\lambda_{3} t}\right) .
$$

Then from Czsisar-Kullback ([13]) inequality we get

$$
\|G-M\|_{L^{1}}=\mathcal{O}\left(e^{-\lambda_{3} t}\right) .
$$

And the estimates $(5.11,5.13)$ lead to (5.2). 


\section{Numerical study.}

In this section we want to illustrate theorem 5.1 by computing in the homogeneous case the $L^{1}$-convergence to equilibrium for different types of molecular interactions and different models -namely the BGK, the ES-BGK and the true Boltzmann equation. Remark that it does not exists a single Boltzmann equation for a chosen type of molecular interaction (see e.g [18, 29, 8, 15]). Moreover solving such an equation requires to use Monte-Carlo method due to its prohibitive deterministic computational cost [4]. For such method it is neither possible to estimate a $L^{1}$-convergence nor to compute a relative entropy. For those reasons we shall consider only monatomic gases. Now let us recall in this case different modelings:

1. the BGK model (we divide it by the molecular mass $m$ as for the Boltzmann collision operator)

$$
R_{B G K}=\frac{\rho}{m}(\tilde{\mathcal{M}}(v)-f(t, v))
$$

with $\tilde{\mathcal{M}}$

$$
\tilde{\mathcal{M}}(v)=\frac{\rho}{(2 \pi R T)^{3 / 2}} \exp \left(-\frac{|v-u|^{2}}{2 R T}\right) .
$$

For the sake of simplicity we still denote $\tilde{\mathcal{M}}$ by $\mathcal{M}$.

2. The ES-BGK model

$$
R_{E S-B G K}=\frac{\rho R T}{\mu(1-\nu)}(G-f)
$$

where $G$ is defined by (4.2) together with the relations $(2.4,2.3,2.6$, $3.8)$,

3. the Boltzmann collision operator

$$
R_{B E}=\frac{1}{m} \int_{\mathbb{R}^{3} \times \mathcal{S}_{+}^{2}}\left(f^{\prime} f_{*}^{\prime}-f f_{*}\right) B(\theta, g) d \theta d \varepsilon d v_{*},
$$

with

$$
v^{\prime}=v-n(n \cdot g), v_{*}^{\prime}=v_{*}-n(n \cdot g), g=v-v_{*} .
$$

$n$ is the unit vector such that

$$
g \cdot n=\frac{\cos (\theta)}{|g|}
$$


and the integral over the half sphere means $g \cdot n>0$. Finally $B(\theta, g)$ is the cross section.

It is well known that the BGK operator was designed for Maxwellian molecules that is when $B(\theta, g)=B(\theta)$. Here we propose to replace this operator with

$$
\tilde{R}_{B G K}=\frac{\rho R T}{\mu}(\mathcal{M}(v)-f(v))
$$

This model will be discussed later on (see section 6.3).

We choose to compute the relaxation time for the argon $(R=208)$ with two different initial data and two different cross sections. The initial data are

1. an anisotropic Gaussian function

$$
f(0, v)=f_{1}(v)=\frac{\rho}{\left(2 \pi R T_{0}\right)^{\frac{3}{2}}} \exp \left(-\frac{\frac{v_{x}^{2}}{2}+v_{y}^{2}+2 v_{z}^{2}}{2 R T_{0}}\right)
$$

with $\rho \simeq 10^{-5} \mathrm{~kg} / \mathrm{m}^{3}$ and $T_{0}=234 \mathrm{~K}$. Then

$$
u=\frac{1}{\rho} \int f_{1}(v) v d v=0 \mathrm{~m} / \mathrm{s}, T=\frac{1}{3 \rho R} \int|v-u|^{2} f(v) d v=273 K .
$$

2. the sum of two Maxwellian functions

$$
\begin{aligned}
f(0, v)=f_{2}(v) & =\frac{\rho}{2}\left(\frac{1}{\left(2 \pi R T_{1}\right)^{\frac{3}{2}}} \exp \left(-\frac{\left(v_{x}-400\right)^{2}+v_{y}^{2}+v_{z}^{2}}{2 R T_{1}}\right)\right. \\
& \left.+\frac{1}{\left(2 \pi R T_{2}\right)^{\frac{3}{2}}} \exp \left(-\frac{v_{x}^{2}+v_{y}^{2}+v_{z}^{2}}{2 R T_{2}}\right)\right)
\end{aligned}
$$

with $T_{1}=30 \mathrm{~K}$ and $T_{2}=300 \mathrm{~K}$. Then

$$
u_{x}=200 \mathrm{~m} / \mathrm{s}, u_{y}=u_{z}=0 \mathrm{~m} / \mathrm{s}, T=229.1 \mathrm{~K} .
$$

We choose to do all computations using discrete velocity models (DVM). This enables to compute the $L^{1}$-convergence and even the relative entropy which is not possible with DSMC. For the BGK and the ES-BGK numerical method we just use a simple Euler scheme. Recall that one can compute the exact solution for the homogeneous Boltzmann equation and that implicit schemes were designed by L.Mieussens $([22,23])$ for those models. The reasons for this are one that we require accuracy in time and two because DVM for the Boltzmann equation do not enable implicit scheme. In this case we refer to $[16,31]$ for the computation of the quadratic integral collision operator. Comparisons with other quadrature formula such as $[28,21]$ give the same results. Now we are going to treat the case of Maxwellian molecules and Hard Spheres. 


\subsection{Maxwellian Molecules.}

In this case the cross section in the Boltzmann collision operator depends only on $\theta: B(\theta, g)=\beta(\theta)$. We choose to take $\beta(\theta)=\cos (\theta)$ which is well suited for the quadrature formula $([16])$. Then the viscosity can be easily computed ([11]) and $\mu=2 k T$. Comparing the different models it is easy to see that a factor $\frac{\rho^{2}}{m}$ appears in $(6.5,6.3,6.4)$. Hence we can define a rescaled time with $t^{\prime}=\frac{\rho}{m} t$.

For the numerical calculations, we use $20 \times 20 \times 20$ velocities with bounds $[-1000,1000]^{3}$ (in $\left.\mathrm{m} / \mathrm{s}\right)$.

Figures 1 and 2 show the $L^{1}$-convergence to equilibrium with initial data $f_{1}$ and $f_{2}$. The main feature in both test cases is the exponential decay of $\|f-\mathcal{M}\|_{L^{1}}$ for each model (time in logarithmic scale). First of all this illustrates Theorem 5.1 for the ES-BGK model but it is also the result proved in $[3,35]$ for the homogeneous Boltzmann equation for Maxwellian molecules with cut-off (the velocity domain is bounded).

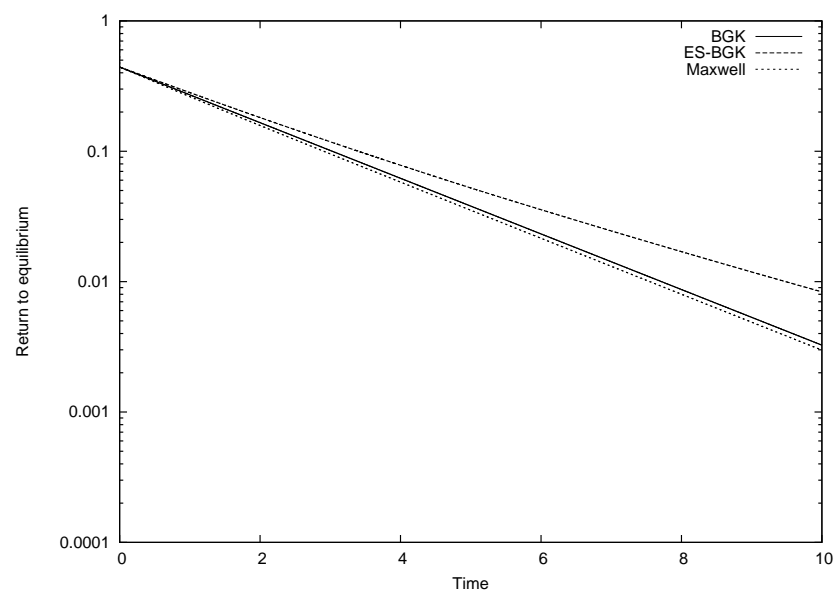

Figure 1: $L^{1}$-convergence to equilibrium.

Comparisons between the rates of relaxation of $\|f-\mathcal{M}\|_{L^{1}}$ and of the relative entropy $H(f \mid \mathcal{M})$-e.g with initial data $f_{1}$, see figure 3 - for the ES-BGK and Boltzmann collision operators illustrate perfectly the Csiszàr-Küllback inequality (5.10) and show that it is quite optimal. This is an important tool that is used in Theorem 5.1 for the ES-BGK model. As concerns the Boltzmann equation the same inequality is used but the essential result is the inequality

$$
H\left(Q^{+}(f, f)\right) \leq H(f)
$$




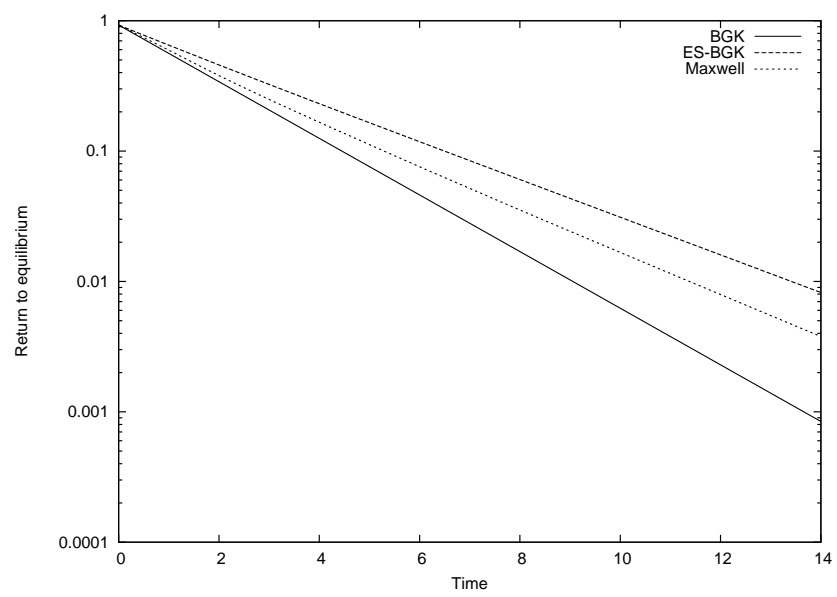

Figure 2: $L^{1}$-convergence to equilibrium.

for Maxwellian molecules. This result was proved by C.Villani ([34]). Again the difference between the slopes fits perfectly with the theory.

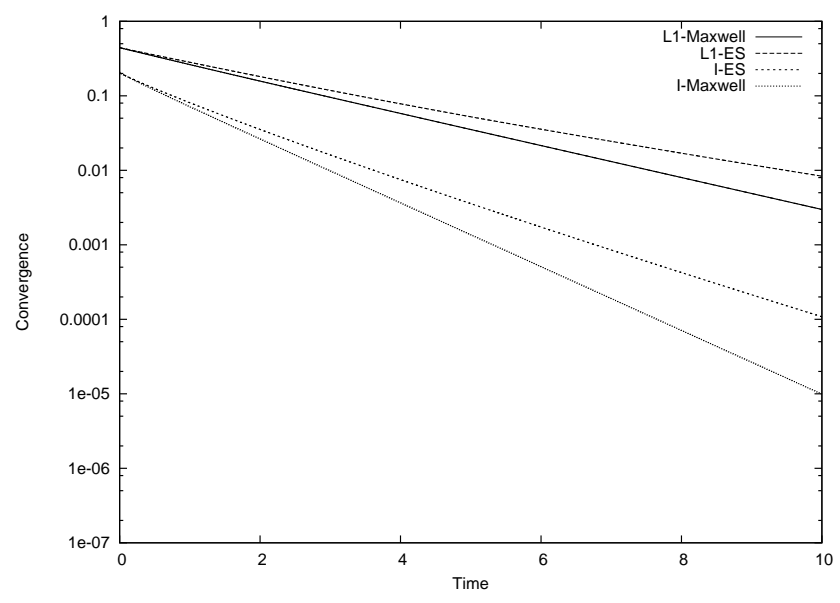

Figure 3: $L^{1}$-convergence and relative entropy.

\subsection{Hard Sphere.}

In this case the cross section $B$ is equal to $\sigma^{2}\left|\left(v-v_{*}\right) \cdot n\right|$ where $\sigma$ is the molecular diameter.

In this context it is proved in [12] that the viscosity $\mu$ is given as a first 
approximation by the expression

$$
\mu=\frac{5}{16 \sigma^{2}}\left(\frac{k m R T}{\pi}\right)^{\frac{1}{2}}=\frac{5 m}{16 \sigma^{2}}\left(\frac{R T}{\pi}\right)^{\frac{1}{2}} .
$$

Hence

$$
\frac{\rho R T}{\mu}=\frac{5}{16} \rho \frac{\sigma^{2}}{m}(R T \pi)^{\frac{1}{2}} .
$$

Recall that in the hard sphere case the Boltzmann equation is given by

$$
R_{B E}=\frac{\sigma^{2}}{m} \int_{\mathbb{R}^{3} \times \mathcal{S}^{2}}\left|\left(v-v_{*}\right) \cdot n\right|\left(f^{\prime} f_{*}^{\prime}-f f_{*}\right) d v_{*} d n .
$$

where $n$ belongs to the whole unit sphere. Hence one can define the rescaled time $t^{\prime}=\frac{\rho \sigma^{2}}{m} t$.

Figures 4 and 5 show the $L^{1}$-convergence to equilibrium with initial data $f_{1}$ and $f_{2}$ in the hard-sphere case. Since there is still a cut-off, the result proved in $[3,35]$ remains valid and decay of $\|f-M\|_{L^{1}}$ in time is still exponential. Finally figure 6 shows the the rates of relaxation of $\|f-\mathcal{M}\|_{L^{1}}$ and of the

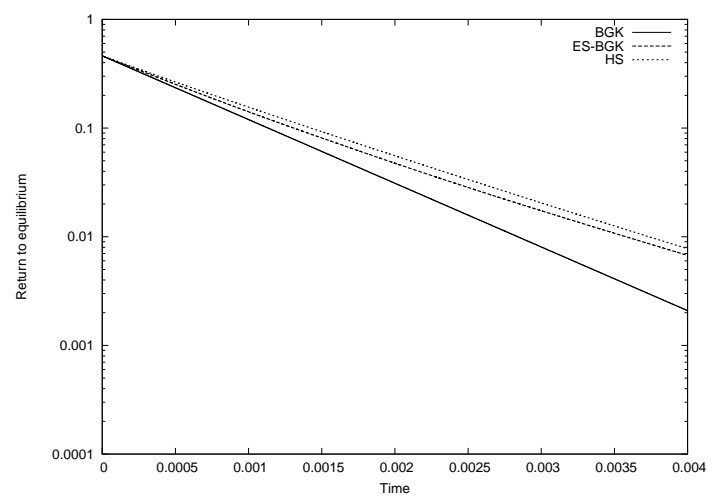

Figure 4: $L^{1}$-convergence to equilibrium.

relative entropy $H(f \mid \mathcal{M})$ with initial data $f_{1}$. In this case the estimate (6.6) is no longer valid but the convergence rates still show the importance of the Csiszàr-Küllback inequality.

\subsection{Discussion.}

Remark that if we use the classical BGK model (6.1) we do not recover the true viscosity. The reason for this is quite simple. Let us write the $B G K$ 


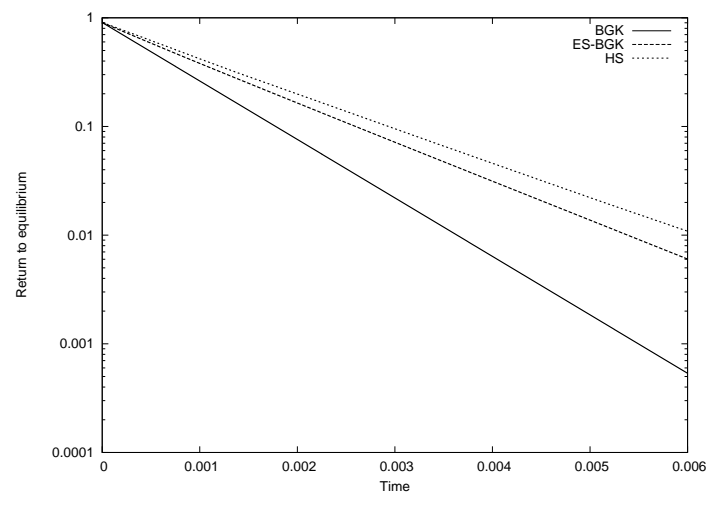

Figure 5: $L^{1}$-convergence to equilibrium.

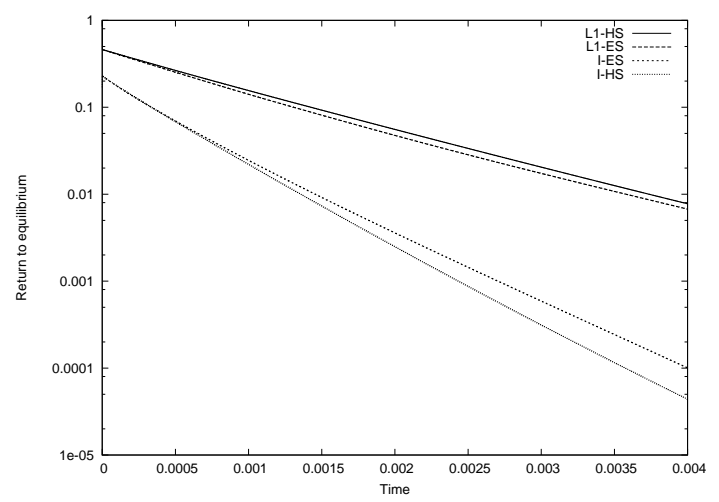

Figure 6: $L^{1}$-convergence to equilibrium. 
model as a relaxation operator of the form

$$
R_{B G K}=\frac{1}{\tau}(M-f)
$$

where $\tau$ is the relaxation rate. Then using the Chapman-Engskog expansion we remark that this relaxation rate appears only in the space derivatives and the viscosity is given by

$$
\mu=\tau \rho R T=k T \text {. }
$$

This viscosity is not far from that obtained with the modified BGK model (6.5) for Maxwellian molecules: $\mu=2 k T$. Remark however that the rate of the $L^{1}$-convergence to equilibrium obtained with the second model fits better with the Boltzmann equation. But for other type of molecular interaction the classical $B G K$ model is not well adapted to any kind of computations. With the modified $B G K$ model one recovers the true viscosity $\mu$ though this model suffers the same deficiency as the classical one: the Prandtl number $\operatorname{Pr}=\frac{5}{2} \frac{R \mu}{\kappa}$ is equal to 1 contrarily to physical case where $\operatorname{Pr} \simeq \frac{2}{3}$. Nonetheless the convergence slopes are approximatively of the same order as the ES-BGK model (up to the factor $\frac{1}{(1-\nu)}=\frac{2}{3}$ ).

Observe that the modified BGK model and the ES-BGK model are essentially constructed -beside classical properties such as conservation laws and $\mathrm{H}$-theorem- so as to give the proper viscosity (and heat conduction coefficient in the second case) for small Knudsen number. This means that there convergence rates to equilibrium is connected with the behavior of the moments $\int f A(V)$ (respectively $\int f B(V)$ ). It is clear for the Boltzmann equation that the behavior of those moments is not the main factor of the convergence rate to equilibrium except when the solution is closed to equilibrium. In this case the analysis of the spectrum of the linearized operator $\mathcal{L}$ shows the importance of those moments ([11]). But in the cases we have chosen to treat, all convergence slopes show an important correlation with all models and henceforth with the above moments.

\section{References}

[1] P.Andries-J.F.Bourgat-P.LeTallec-B.Perthame Numerical comparison between the Boltzmann and the ES BGK models for rarefied flows. Comp.Meth.Appl.Mech.Eng. 191 (2002), 3369-3390.

[2] P.Andries-P.LeTallec-J.P.Perlat-B.Perthame Entropy condition for the ES BGK model of Boltzmann equation for mono and polyatomic gases. European J. Mechanics (B fluids) 19, 813-830 (2000). 
[3] L.Arkeryd Stability in $L^{1}$ for the spatially homogeneous equation. Arch.Rat.Mech.Anal. 103 (2), 151-167, 1988.

[4] Y.Bahi Contribution la simulation numrique des coulements en gaz rarfis. Phd Thesis, University Pierre et Marie Curie, Paris, 1997.

[5] P.L.Bhatnagar-E.P.Gross-M.Krook. A model for collision processes in gases. Phy.Rev., 94-511, 1954.

[6] C.Borgnakke-P.S.Larsen-Statistical collision model for Monte-Carlo simulation of polyatomic gas mixtures J.C.P., 18, 405-420, 1975.

[7] F.Bouchut-B.Perthame. A BGK model for small Prandtl number in the Navier-Stokes approximation. J.Stat.Phys., 71(1-2):191-207, 1993.

[8] J.F.Bourgat-L.Desvillettes-P.Le Tallec-B.Perthame Microreversible Collisions for Polyatomic Gases and Boltzmann's Theorem, European Journal of Mechanics, B/ Fluids, vol. 13, n. 2, (1994), pp. 237-254.

[9] S.Brull-J.Schneider A new approach of the Ellipsoidal Statistical Model. Cont.Mech.Thermodyn. 20, no.2, 63-74, 2008.

[10] R.Brun Transport et relaxation dans les écoulements gazeux. Masson Paris 1986.

[11] C.Cercignani. The Boltzmann equation and its applications. Scottish Academic Press, 1988, 40-103.

[12] S.Chapman-T.G.Cowling. The Mathematical Theory of non-uniform gases. Cambridge Mathematical Library, third edition, 1970.

[13] I.Csiszár. I-divergence geometry of probability distributions and minimization problems Sanov property. Ann.Probab 3, 146-158, 1975.

[14] J.F.Collet Extensive Lyapounov functionals for moment-preserving evolution equations. C.R.A.S. Ser.I 334 (2002) p. 429-434.

[15] L.Desvillettes. Sur un Modèle de type Borgnakke-Larsen Conduisant des lois d'Energie Non-linaires en Température pour les Gaz Parfaits Polyatomiques, Annales de la Faculté des Sciences de Toulouse, Série 6, vol. 6, n. 2, pp. 257-262, 1997.

[16] D.Goldstein-B. Sturtevant and J. E. Broadwell. Investigation of the motion of discrete-velocity gases, in "Rarefied Gas Dynamics: Theoretical 
and Computational Techniques", E. P. Muntz, D. P. Weaver and D. H. Campbell Eds., Progress in Astronautics and Aeronautics 118, 100-117, 1989.

[17] L.H.Holway Kinetic theory of shock structure using an ellipsoidal distribution function. In rarefied Gas Dynamics, vol I (Proc. Fourth Internat. Sympos. Univ. Toronto, 1964) Academie Press, New York, 1966 193-215.

[18] I.Kusker A model for rotational energy exchange in polyatomic gases Physica A 158, 1989 784-800.

[19] P.Le Tallec, A hierarchy of hyperbolic models linking Boltzmann to Navier Stokes equations for polyatomic gases, ZAMM, 80,11-12, 779-790, 2000.

[20] C.D.Levermore. 'Moment closure hierarchies for kinetic theories', Journ.Stat.Phys. 831996 1021-1065.

[21] P.Michel-J.Schneider. Approximation simultanée de réels par des nombres rationnels et noyau de collision de l'équation de Boltzmann. CRASsérie 1 maths.330 (2000) n9, 857-862.

[22] L.Mieussens. Modèles à vitesses discrètes et méthodes numériques pour l'équation de Boltzmann BGK. Phd Thesis 1999.

[23] L.Mieussens. Discrete velocity models and implicit scheme for the BGK equation rarefied gas dynamic. M3AS 10, 2000, n8, 1121-1149.

[24] L.Mieussens-H.Struchtrup. Numerical comparison of BGK-models with proper Prandtl number, Phys.Fluids 16(8), 2797-2813 (2004)

[25] M.Junk Domain of definition of Levermore's five-moment system. J. Stat. Phys., Vol 93, 1143-1167, 1998.

[26] M.Junk 'Maximum entropy for reduced moment problems', M3AS 10 2000 1121-1149.

[27] E.M.Lifschitz, L.P.Pitaevskii Physical kinetics, Pergamon Press., Oxford 1981

[28] F.Rogier-J.Schneider $A$ direct method for solving the Boltzmann equation. TTSP 23 (1994) n1-3, 313-338.

[29] D.I.Pullin Kinetic models for polyatomic molecules with phenomenological energy exchange Phys.Fluids, (1978) 21, 209-216 
[30] J.Schneider 'Entropic approximation in kinetic Theory', M2AN, vol.38 no3, 2004 541-561.

[31] A.Palczewski-J.Schneider-A.Bobylev. A consistency result for a discrete-velocity model of the Boltzmann equation. SIAM.J.Numer.Anal. (1997) vol 34, No5, 1865-1883.

[32] H.Struchtrup 'The BGK model with velocity dependent collision frequency' Cont.Mech.Thermodyn. 9, 23-31 (1997)

[33] G.Toscani 'Remarks on entropy and equilibrium states.' Appl.Math.Lett.12 (7) (199) 19-25.

[34] C.Villani Fisher information estimates for Boltzmann's collision operator.' J. Maths Pures Appl. 77 (1998), 821-837.

[35] B.Wennberg 'Stability and exponential convergence for the Boltzmann Equation' Phd thesis, Chalmers Univ. Tech., 1993.

\section{APPENDIX A: Proof of lemma 4.1.}

For any function $h \in L_{1}^{2}$ consider the function $g_{\alpha, \beta}$ defined with

$$
g_{\alpha, \beta}=\frac{1}{\mathcal{I}_{\alpha, \beta}} \frac{1}{\operatorname{det}(\chi)^{\frac{1}{2}} \beta^{\frac{\delta}{2}}} h\left(-\alpha\left\langle v-u, \chi^{-1}(v-u)\right\rangle-\beta I^{\frac{2}{\delta}}\right)
$$

with

$$
\mathcal{I}_{\alpha, \beta}=\frac{1}{\rho} \int_{\mathbb{R}^{3} \times \mathbb{R}_{+}} \frac{1}{\operatorname{det}(\chi)^{\frac{1}{2}} \beta^{\frac{\delta}{2}}} h\left(-\alpha\left\langle v-u, \chi^{-1}(v-u)\right\rangle-\beta I^{\frac{2}{\delta}}\right) d v d I .
$$

Then one easily see that $g_{\alpha, \beta}$ has the same mass and momentum as $f$. Next, in order to get the relation

$$
\int_{\mathbb{R}^{3} \times \mathbb{R}_{+}} I^{\frac{2}{\delta}} g_{\alpha, \beta} d v d I=\frac{\delta}{2} \rho R T_{r e l}
$$

we put $\beta^{\frac{\delta}{2}}=\frac{\delta}{2} \rho T_{r e l}$. Check that the constraint w.r.t. $\frac{|c|^{2}}{2}+I^{\frac{2}{\delta}}$ is also satisfied. The fourth relation of (4.1) is equivalent to

$$
\int_{\mathbb{R}^{3} \times \mathbb{R}_{+}} \frac{|c|^{2}}{2} g_{\alpha \beta} d v d I=\frac{3+\delta}{2} \rho R T_{e q}-\frac{\delta}{2} \rho R T_{r e l} .
$$


$\chi$ being being symmetric definite and nonnegative such that $\chi=S^{2}$.

$$
\begin{array}{r}
\int_{\mathbb{R}^{3} \times \mathbb{R}_{+}}|S c|^{2} h\left(-\alpha|c|^{2}-\beta I^{\frac{2}{\delta}}\right) d v d I=\frac{\operatorname{tr}(\chi)}{\alpha^{\frac{5}{2}}} \int_{\mathbb{R}^{3} \times \mathbb{R}_{+}}\left|S c_{1}\right|^{2} h\left(|c|^{2}-\beta I^{\frac{2}{\delta}}\right) d v d I, \\
\mathcal{I}_{\alpha, \beta}=\frac{1}{\rho \alpha^{\frac{3}{2}}} \int_{\mathbb{R}^{3} \times \mathbb{R}_{+}} h\left(-|c|^{2}-\beta I^{\frac{2}{\delta}}\right) d c .
\end{array}
$$

By choosing $\alpha$ such that

$$
\alpha=\frac{2}{3} \frac{\operatorname{Tr}(\chi)}{T_{r e l}} \frac{\int_{\mathbb{R}^{3} \times \mathbb{R}_{+}}\left|c_{1}\right|^{2} h\left(-|c|^{2}-\beta I^{\frac{2}{\delta}}\right) d c d I}{\int_{\mathbb{R}^{3} \times \mathbb{R}_{+}} h\left(-|c|^{2}\right) d c d I}
$$

the fourth relation of (4.1) is obtained. Therefore $\mathcal{C}_{f} \neq \emptyset$. 